\title{
LA DIFERENCIA ÉTNICA CONSTRUIDA POR EL ESTADO: IDENTIDAD NACIONAL MEXICANA E IDENTIDAD INDÍGENA ${ }^{1}$
}

\author{
Eva Sanz Jara
}

L a imagen que el Estado tiene de sí constituye el proyecto nacional. El Estado considera a los indígenas población diferenciada, a la vez los asume como mexicanos, por ello necesita considerarlos para definirse. Los indígenas, al formar parte de la nación, también integran el proyecto nacional. El Estado ha diseñado a lo largo de los siglos XIx y xx varios modelos de nación, al variar la autopercepción, la manera de tomar en cuenta la diferencia también ha cambiado. En el proyecto nacional vigente en cada momento se les asigna un lugar a los indios, dicho lugar se explicita a través de la definición de indígena que el Estado ofrece, en la que se incluye cierto otorgamiento de identidad. En otras palabras, el Estado asigna a los indígenas parte de su identidad para que ésta responda a las necesidades estatales de autopercepción, modelando así identidades ${ }^{2}$ en función del momento histórico con la finalidad de construir el proyecto nacional.

El Estado mexicano contribuye a definir lo indígena mediante el discurso que emite sobre el término. Este discurso se difunde y simultáneamente se construye a través de varios medios que detenta: censos de población, discurso político, legislación, y la producción intelectual. Este artículo se ocupa de los censos. En

Eva Sanz Jara, estudiante del Programa de Doctorado en América Latina Contemporánea del Instituto Universitario de Investigación Ortega y Gasset. ellos, el Estado comunica su definición de indígenas mediante la asignación de categorías. Categorizar supone un ejercicio de poder, pues significa clasificar en diferentes grupos a la población, asignando con ello, en gran medida, identidades que condicionan la autoimagen de los individuos y, por extensión, sus acciones.

Existen dos grandes corrientes referidas a la cuestión identitaria, la primordialista y la instrumentalista (Chihu, 2002). La primera, también denominada esencialista u objetivista, concibe la identidad como esencia, como algo natural, objetivo, inevitable para el individuo, con raíces históricas e incluso biológicas. Por su parte, la corriente instrumentalista, constructivista o subjetivista, define la identidad como construcción social, basada en rasgos subjetivos, que persigue la consecución de determinados fines.

La identidad es un término que combina semejanza y diferencia: semejanza con el grupo de pertenencia y diferencia respecto a los otros. Admitir que existen otros es el germen de la identidad, por definición es relacional; este reconocimiento de la otredad se lleva a cabo mediante la destotalización. ${ }^{3} \mathrm{~L}$ a identidad es negativa, pues sus rasgos se definen por oposición a los de los otros (Chihu, 2002; Giménez, en Chihu, 2002; Hernando, 2002).

La identidad es construida en un proceso donde de entre todos los rasgos que en primera instancia se poseen, se seleccionan algunos de ellos: son los que 
sufren las modificaciones necesarias para hacerlos operativos, así se convierten en estereotipos. El proceso de construcción de la identidad se realiza trazando fronteras entre el grupo y el resto, la exageración de las características definitorias tiene como finalidad que dichas fronteras sean sumamente nítidas; frecuentemente ocurre que las fronteras las trazan los grupos dominantes para separarse de los demás.

La identidad es subjetiva, puesto que se basa en rasgos seleccionados y modificados en función de los intereses de quien lleva a cabo el proceso de construcción de la identidad. No obstante, subjetividad no significa arbitrariedad.

La identidad es situacional, no cualquier identidad es posible, debe tener cabida dentro de la sociedad, el momento histórico y las corrientes políticas vigentes.

Las identidades de los individuos se dividen en categorías, a las que dichos individuos se adscriben o son adscritos desde el exterior. Estas categorías no son fijas ni estáticas, varían con el tiempo para adaptarse a las exigencias de los diferentes momentos (Bartolomé, 1997; Chihu, 2002; Pujadas, 1993). La identidad de las personas no es única, pero una de ellas domina, al menos en cada momento; no se manifiestan al mismo tiempo varias identidades igual de relevantes (Hobsbawm, 1994).

La perspectiva constructivista es mayoritaria en el mundo académico, mientras que la primordialista domina tanto la concepción política — del Estadocomo la popular. De la adscripción a la corriente constructivista o instrumentalista se desprenden algunas premisas: las categorías identitarias son construidas no sólo por los propios grupos, en su creación participa también el Estado que, teniendo en cuenta el momento histórico y la ideología vigente, permite ciertas identidades y no otras; además es necesario que el Estado "nombre" —instaure- dichas identidades para validarlas. Por último, se parte de la afirmación de que las categorías identitarias no son arbitrarias, responden a ciertas motivaciones, a ciertos intereses.
Se propone que la identidad es modelada por el Estado, o al menos seleccionada entre otras que los grupos sociales son susceptibles de construir. Quién realiza la categorización es la cuestión fundamental; determinar que las categorías no las crean en su totalidad los grupos y que los individuos no se adscriben voluntariamente a ellas sino que, por el contrario, quien detenta el poder juega un papel fundamental en esta asignación identitaria, supone la cuestión nuclear en este escrito. Insertar en categorías a la población es una forma suprema de ejercer el poder, puesto que en función de la identidad que se confiere a los individuos, o que se les permite que adquieran, se establece la imagen de sí mismos que van a poseer, y ésta va a condicionar sus actuaciones en todos los ámbitos.

La identidad nacional surge como modo de cohesión creado por las élites debido a la heterogeneidad característica de todas las poblaciones nacionales. Dicha heterogeneidad es la causa de la creación del nacionalismo, elemento que legitima la homogeneización de la diferencia (Anderson, 1983). Constituye la hipótesis de este artículo que la identidad étnica está fuertemente ligada a la identidad nacional, debido a que la primera ha sido modelada por el Estado mexicano para construir distintos proyectos de nación a lo largo de los siglos XIX y Xx.

Con el nacimiento de México como nación se pone en marcha, por parte del Estado, el "proyecto liberal", vigente durante todo el siglo XIX, que tiene como propósito, en lo que se refiere a los indígenas, darles el tratamiento opuesto al que recibieron durante la Colonia: si los españoles trataron de mantener apartado al indio del resto de la sociedad, el objetivo del "proyecto liberal" será el de la asimilación, dado que se considera la pervivencia del indio un obstáculo al progreso. La asimilación significa terminar con lo diferente, homogeneizar la nación entera, lo que conlleva necesariamente la desaparición de la cultura indígena. 
El indio no va a jugar un papel importante en el proyecto liberal, porque admitir la diferencia hubiera implicado cuestionar la idea de nación, que en ese momento se basaba en la ciudadanía igualitaria. Para lograr la igualdad deseada abolieron las formas jurídicas diferenciatorias imperantes en el periodo anterior. No obstante, las relaciones sociales no varían nada más producirse la Independencia, ya que están firmemente enraizadas desde siglos atrás; de hecho, muchas de ellas, como la discriminación de que son objeto los indígenas en la práctica, permanecerán hasta nuestros días.

Con la Revolución de 1910 la ideología del mestizaje se convierte en proyecto nacional. En este contexto nace el indigenismo, que toma lo indio como componente básico de la nación. El indígena en la ideología del mestizaje es un concepto que se desdobla: por un lado está el indio prehispánico, sobrevalorado por ser parte integrante del actual mestizo, por otro el indígena contemporáneo, subvalorado porque impide la existencia de una nación homogénea y desarrollada. $\mathrm{El}$ "proyecto indigenista" propone la integración del indio en el Estado mexicano para conseguir el desarrollo, tanto de la sociedad en general como de los propios indígenas; para lograr este objetivo se debe, primero, rescatar la herencia cultural indígena prehispánica, segundo, posibilitar que el indio abandone todas las características que le mantienen en un estado de subdesarrollo. El "periodo indigenista" abarca desde la década de los 20 hasta la de los 90, momento en que es sustituido por un nuevo paradigma, tras haber sido objeto de fuertes críticas desde finales de la década de 1960.

Las críticas al integracionismo desembocarán en una crisis que terminará con el "proyecto indigenista". Más tarde se abre paso un nuevo proyecto encabezado por los principales críticos del indigenismo: los antropólogos y líderes de organizaciones indígenas; pero no sólo es gracias a estos actores que el nuevo proyecto logra instaurarse, la coyuntura internacional de la globalización juega un papel decisivo. México decide asumir su heterogeneidad, no para integrarla sino para conservarla, comenzando así un periodo de valoración de la diferencia que perdura hasta la actualidad y que puede denominarse "proyecto pluralista". No obstante, es necesario señalar que el objetivo último sigue siendo el mismo que en el momento de la Independencia: la consecución del proyecto de una Nación mexicana.

\section{Los indígenas en los censos de población mexicanos}

Al trabajar con censos de población es conveniente comenzar por cuestionar la razón de ser del objeto de estudio: ¿por qué existen los censos?, ¿por qué es necesario contar a la población? Estos porqués se responden si se contesta, a su vez, a dos cuestiones: ¿quiénes realizan los censos?, ¿con qué finalidad lo hacen? E. Malvido y M.A. Cuenyas ${ }^{4}$ responden que quienes cuentan detentan el poder y censar constituye una forma de ejercerlo. Contabilizar a la población supone obtener información necesaria para llevar a cabo un gobierno efectivo sobre ella. No sólo contar significa poseer poder, clasificar implica ser aún más poderoso.

Los censos de población constituyen la principal fuente de información demográfica de un país, son raramente cuestionados debido a que los datos que ofrecen aparecen con cifras, se tiende a sobrevalorar la información numérica. Resulta difícil afirmar que los datos censales no son imparciales porque se tiende a pensar que la información cuantitativa está exenta de toda ideología. Tradicionalmente, el trabajo con censos de población se reducía a extraer conclusiones a partir de dar por ciertas las cifras que en ellos aparecían, sin cuestionarlas en absoluto. Este escrito se ha guiado por autores $^{5}$ que sí cuestionan estas fuentes. Los datos censales siempre tienen una desviación, eso es lo que los hace especialmente relevantes, porque interpretando dicha desviación y deduciendo sus motivos se pueden relacionar con la coyuntura histórica en que fueron 
levantados y con la ideología imperante durante su realización. ${ }^{6}$

A través de los censos, el Estado categoriza a la población. Según D. Yanow, las categorías sirven para ordenar el mundo y son propias de los humanos y de Dios -Dios puso nombre, categorizó, todo lo existente en el cielo y en la tierra-, luego la categorización es una actividad que requiere de mucho poder para ser ejercida. Categorizar significa asignar diferenciación e identificación a determinados elementos - en este caso a las personas-, pero no se lleva a cabo de manera arbitraria, sino que se hace en función de las circunstancias históricas que rodean a los "categorizadores", en palabras de la autora: "Las categorías reflejan un momento histórico con sus "realidades pertinentes" (Yanow, en Greenwood y Greenhouse, 1998: 181).

Teniendo en cuenta que se establecen en función del momento histórico, las categorías no son estáticas, se tiende a tratarlas como si lo fueran porque supuestamente reflejan fenómenos naturales, lo que implica que los "categorizados" no pueden zafarse del lugar que se les destina. Las categorías no pueden ser fijas en ningún caso, puesto que dependen del momento en que se realizan y del criterio de los "categorizadores", que iluminan unos rasgos y oscurecen otros en función de sus intereses: "Las categorías del censo no fueron creadas para permitir que los individuos expresen sus identidades. Tenían propósitos instrumentales claros [...]" (Yanow, en Greenwood y Greenhouse, 1998: 208).

Los conjuntos de categorías toman como referente la categoría "normal" - hombre blanco, idioma inglés, en Estados Unidos; hombre mestizo, idioma español, en América latina-y, a continuación, se va ordenando la "otreidad" en función de esa "normalidad". "Blanco" o "mestizo" son categorías vacías, no tienen características determinadas, constituyen el baremo a partir del cual se juzga al resto, a los "otros". Todo lo que se salga del prototipo significa "otreidad", minoría, desviación.

Las categorías censales pueden dividirse en incluyentes y excluyentes. "Negro" es ejemplo paradigmático de categoría en las primeras, mientras que "indio" lo es de las segundas.7 Los Estados que utilizan categorías incluyentes buscan diferenciar a las poblaciones categorizadas; mientras que los que usan categorías excluyentes persiguen que las poblaciones englobadas en esa categoría abandonen su identidad y se integren a la mayoritaria.

Censar consiste en contabilizar a la población insertándola en diferentes categorías, pero como éstas no son naturales se tiende a englobar bajo categorías homogeneizantes a grupos sumamente heterogéneos. Prueba de que las categorías no son esenciales es el hecho de que se utilizan diferentes criterios para definirlas.

No todos los Estados americanos utilizan los mismos criterios de identificación de los indígenas, existen visibles discrepancias entre ellos. "El problema principal radica en la propia definición de población indígena, tanto en lo conceptual como en el indicador operativo para identificarla en los censos y encuestas" (Peyser y Chackiel, en CELADE, 1994: 28). No existe consenso en la definición del término "indígena" y tampoco hay acuerdo en lo referente a los criterios que definen a dichas poblaciones; esto acarrea problemas en el conteo. Los criterios clasificatorios establecen qué significa ser indígena y pretenden ser el medio para contar su número total, así como su ubicación geográfica y comportamiento demográfico; éstas cuestiones han suscitado debates y polémicas entre los intelectuales estudiosos del tema indígena desde hace décadas.

No existe acuerdo a la hora de definir a los indígenas actuales, pero sí al establecer el origen del término. "Indígena" nace a partir del concepto "indio", puesto que se utiliza la denominación "indígena" para los pueblos que estaban en el actual territorio nacional antes de la llegada de los europeos; e "indios" es la 
denominación que los españoles de hace 500 años, al llegar al continente americano, dieron a los habitantes originarios, dando con ello comienzo a la homogeneización y a la discriminación que, en muchos aspectos, continúa en la actualidad bajo ambos aspectos.

Tras la Independencia de México el término "indio" deja de ser utilizado, al menos oficialmente, y se pasa a denominar a estas poblaciones "indígenas". No obstante, es necesario señalar que el asunto es bastante más complejo. Cuando los colonizadores, al inicio de la Conquista, llamaron "indios" a los pobladores del Nuevo Continente hacían referencia a poblaciones fenotípicamente diferenciadas; pero, tres siglos de colonia y dos de vida independiente, teniendo en cuenta el mestizaje ocurrido durante todo ese tiempo, no existen rasgos físicos que distingan claramente a ambas poblaciones y, en cuanto a los rasgos culturales, la aculturación acaecida hace que la diferenciación sea también difusa.

Pese a lo dicho con anterioridad, es idea generalizada que la población mexicana puede dividirse en dos grandes grupos: indígenas y no indígenas, a pesar de que, ahora, finalizado el siglo xx: "Es imposible trazar una línea definida de demarcación entre la población indígena en conjunto y la población no indígena precisamente porque cada una de ellas ha adquirido rasgos de la otra" (Manrique, 1994: 5).

Es necesario añadir que la población india colonial no se corresponde exactamente con los indígenas del México independiente. Ser indio durante la Colonia tenía numerosas implicaciones, diferentes de las que ha tenido ser indígena desde el siglo XIX hasta la fecha. Fundamentalmente, ser indio durante el periodo colonial, en el que imperaba un sistema que podría denominarse de castas, implicaba formar parte, por nacimiento y de por vida, de una población diferenciada, con las ventajas e inconvenientes que ello conllevaba; en el México de los siglos XIx y xx, al no existir una línea nítida de separación entre la población indígena y la que no lo es, las personas pueden en muchos casos identificarse o no como indígenas en función de múltiples condicionantes externos.

Pese a la imposibilidad de diferenciar claramente indígena de no indígena en México, las instituciones a menudo ofrecen explicaciones esencialistas y homogeneizantes acerca de la población étnicamente diferenciada: "Al momento de la conquista en el territorio nacional se localizaban grandes imperios en su plenitud social y cultural; al entrar en contacto con los españoles se inicia un proceso de mestizaje y castellanización. A pesar de esto, en la actualidad algunos núcleos por su número y cohesión grupal han subsistido, al igual que aquellos que habitan en zonas de difícil acceso" (INEGI, 1993: Introducción). El Instituto Nacional de Estadística, Geografía e Informática sólo cita a los "grandes imperios", obviando al resto de indígenas que habitaban en lo que hoy es México; describe la identidad como algo esencial, necesario, afirmando la inevitabilidad de la subsistencia cultural debido al difícil acceso, a la cohesión y al número; y no establece una diferencia entre la supervivencia del grupo y la de la cultura.

La homogeneización artificial mediante la denominación de "indígenas" es, según autores como M. A. Bartolomé (INI, 2000), característica común a la mayoría de los pensadores que han reflexionado sobre la cuestión indígena. La única generalización válida, desde el punto de vista del autor, es la que G. Bonfil establece al calificar a los indígenas como los "colonizados". Explica M. A. Bartolomé que la identidad atribuida homogeneizante ha sido, tras ser continuamente repetida por el Estado, asumida por los propios indígenas: "Surgen así autodefiniciones que en realidad corresponden a los predicados adjudicados a la indianidad genérica" (ibid., p. 28). Tal indianidad genérica es asumida como cierta por todos los criterios utilizados para establecer la categoría “indígena": el criterio racial, el basado en la filiación, la ubicación geográfica, la autoadscripción, el criterio cultural y la lengua hablada. 


\section{Criterios utilizados para definir la categoría "indígena"}

Las categorías que aparecen en los censos no son reflejo de fenómenos naturales, ni siquiera la división por razas de la población que: "Es una construcción social que hemos impuesto al mundo humano" (Yanow, en Greenwood y Greenhouse, 1998: 187). El criterio racial es el más antiguo utilizado para dividir a la población en categorías, pero tiene graves inconvenientes. En primer lugar ocasiona equívocos, puesto que suele confundirse la raza de una persona con su cultura, su religión, su origen geográfico, etc. Además, el mestizaje impide la utilización de este criterio. Por último, "raza" es un concepto que se considera discriminatorio en gran parte del mundo. ${ }^{8}$

La filiación es un criterio similar al racial, aunque existe una diferencia destacable entre ambos: mientras que el criterio racial es incluyente, pues se mide en función del aspecto físico, la filiación es excluyente, ya que necesita de la demostración de ascendencia indígena. Por lo anterior, el criterio racial normalmente implica valoración negativa de la identidad, mientras que la filiación conlleva valoración positiva. La nación que constituye el ejemplo paradigmático de uso del criterio de filiación es Canadá. ${ }^{9}$

La ubicación geográfica es un criterio útil en los países en donde los indígenas se circunscriben a determinados territorios, ${ }^{10}$ pero no sirve para censar a la población indígena del resto de países.

La autoidentificación o autopercepción sobre la pertenencia a un grupo o comunidad indígena tiende a arrojar cifras muy dispares, por lo que suele ser considerado sobreestimador o subestimador, dependiendo de los casos, debido a que son identificadas como indígenas personas que según el resto de criterios no lo serían, de igual manera como no indígenas población que lo sería según los demás criterios. Además, los resultados que arroja se modifican sustancialmente en función del modo en que se haga la pregunta al realizar el conteo.

El autoadscriptivo es un criterio tildado de subjetivista y criticado por ser difícil de operativizar y por prestarse a la instrumentalización ${ }^{11}$ de la identidad, pero lo cierto es que resulta coherente, puesto que las personas actúan de acuerdo con la identidad que declaran. Ahora bien, la diferencia fundamental de este criterio respecto al resto es que si se pone en práctica, el Estado pierde la prerrogativa de clasificar, mediante la otorgación de identidades, a la población, quedando la tarea en manos de los propios individuos.

El criterio cultural es defendido por antropólogos del "periodo indigenista", que están de acuerdo en que el resto de criterios subnumeran u ofrecen una imagen desvirtuada de las poblaciones indígenas. Caso opina que para definir "qué es ser indio" deben combinarse los criterios autoadscriptivo y cultural, ${ }^{12}$ mientras que M. Gamio ${ }^{13}$ afirma que la lengua hablada no es suficiente para clasificar a la población indígena, también deben tenerse en cuenta los criterios racial y cultural.

Pero el criterio cultural ha de ser tratado con sumas reservas porque, si se tiene en cuenta que no existen características culturales “puras", puesto que todos los grupos indígenas mexicanos han mantenido contacto con la cultura occidental y ambas se han influenciado, ${ }^{14}$ ¿cómo establecer qué rasgos culturales son exclusivamente indígenas?

La lengua hablada es el criterio escogido con más frecuencia por los Estados latinoamericanos; se categorizan como indígenas las poblaciones que hablan lenguas amerindias de origen prehispánico. Constituye el criterio preferido porque es, a primera vista, aséptico y "objetivo". Pero bajo este halo de imparcialidad se esconden fuertes polémicas, que no siempre quedan reflejadas en los trabajos producidos por las instituciones encargadas de realizar el censo: "Por ser el criterio lingüístico el indicador más aproximado a la identificación de la población indígena, en este 
documento [censo de 1990] se utilizan indistintamente los términos población indígena y población hablante de lengua indígena" (INEGI, 1993: Introducción). El INEGI, deliberadamente, no establece distinción entre "población hablante de lengua indígena” y "población indígena”, este equívoco puede ser considerado como una de las principales objeciones que pueden hacérsele al criterio lingüístico. Algunos autores le reprochan precisamente lo contrario: ${ }^{15}$ los censos no informan sobre población indígena sino sobre lenguas indígenas. ${ }^{16}$ Varios autores (Peyser y Chackiel, en CELADE, 1994; Valdés y Menéndez, 1987) llaman la atención sobre otro inconveniente que trae consigo la utilización de la lengua como criterio único, y es que no se registra en los censos ningún cambio en las culturas indígenas. La lengua es una característica que se abandona con facilidad, debido a las migraciones o a la educación, por ejemplo, sin que la pérdida de la lengua signifique necesariamente el abandono de la cultura o de la identidad étnica. Categorizar a los indígenas sólo por el idioma implica concebir sus culturas como estáticas.

El hecho de que no exista un criterio universalmente validado se debe a que cada uno de ellos posee sus propios inconvenientes, lo que acarrea numerosas dificultades a la hora de establecer la cifra total de población indígena que habita en un determinado país. ${ }^{17}$ Los criterios identificatorios descritos aparecen en los censos mayoritariamente de manera independiente, aunque, según instituciones como Naciones Unidas, resulta insuficiente utilizar en los censos un solo indicador de identidad indígena porque subestima el número total de la población indígena hasta en $22 \%$ (www.un.org).

La subnumeración de la población indígena es, según muchos de los autores y organismos consultados, característica inherente a los censos desde su origen hoy; la Comisión para el Desarrollo Indígena lo expresa de la siguiente manera: "Existe consenso en reconocer que los datos censales sobre hablantes de lenguas autóctonas representan un límite inferior del número total de indígenas en México" (www.cdi.gob.mx). Dicha subnumeración puede producirse a varios niveles. Se habla de subnumeración censal ${ }^{18}$ cuando son los conteos de población los responsables de la misma; es decir, cuando las preguntas predisponen a respuestas negativas, cuando se dejan de registrar regiones por su aislamiento o su mala accesibilidad, cuando los criterios utilizados hacen que cierto número de indígenas no aparezca como tal, etc. Puede hablarse de subnumeración causada por los propios indígenas cuando parte de ellos decide no identificarse como tal. $Y$ debe tenerse en cuenta un nivel superior de subnumeración, denominado por L. M. Valdés "etnocidio estadístico", ${ }^{19}$ que se produce cuando se obvia a la población indígena de los estudios sociodemográficos nacionales.

En opinión de A. Peyser y J. Chackiel (CELADE, 1994), la información que aparece en los censos de población latinoamericanos sobre sus poblaciones indígenas es escasa y deficiente debido a varias limitaciones, la primera atañe a la dificultad de definir qué es ser indígena, la segunda concierne a la elección del criterio o criterios adecuados para identificar a dicha población, añadiéndose a ellas los prejuicios existentes en estas poblaciones a la hora de identificarse como indígenas. Pero, a pesar de las limitaciones, los censos de población son la principal fuente para determinar la cuantía total de la población indígena.

\section{Los indígenas en los censos mexicanos modernos}

Al lograr México su independencia en el siglo XIx, los políticos ilustrados y liberales promulgan la igualdad de los individuos ante la ley. El término "indio", utilizado durante la Colonia, lleva implícito desigualdad, por lo que deja de usarse tras la Independencia —o, al menos, eso se pretende desde el poder-, siendo sustituido por "indígena". Se termina así con las 
diferencias jurídicas entre los habitantes de México. En la práctica la situación es muy diferente, ya que la estructura social vigente durante la Colonia, basada en la desigualdad, no sólo no desaparece de un día para otro tras el nacimiento de la nación, sino que se perpetúa, desarrollando características nuevas durante el periodo independiente. Al seguir existiendo, en la práctica, una población diferenciada del resto, el Estado se ve obligado a reflejarla en los censos, contradiciendo de este modo sus ideales de igualdad y unidad nacional.

Durante el Porfiriato, el Estado implanta el conteo periódico de población como respuesta al ingreso del país en la órbita del capitalismo mundial y a la modernización del Estado nacional. Estas coyunturas "hacían indispensable el conocimiento de la población, de su composición, de su estructura y de su distribución para de ese modo plantear las necesidades presentes y su proyección futura" (Malvido y Cuenyas, 1993: 22).

El primer censo nacional moderno se lleva a cabo en 1895. Desde ese año hasta hoy, los censos se han venido realizando cada diez años, con dos excepciones. La primera de ellas se produce en 1900, momento en que se realiza un censo con sólo cinco años de distancia respecto al anterior; la segunda tiene lugar en la década de 1920, en ella el censo se lleva a cabo en 1921, debido a la Revolución. Existen, pues, doce censos modernos queinforman de la población mexicana: 1895, 1900, 1910, 1921, 1930, 1940, 1950, 1960, 1970, 1980, 1990 y 2000.

La proporción de población indígena respecto al total nacional que aparece en los censos acusa fuertes altibajos. Manrique apunta que las variaciones no responden a fenómenos de carácter demográfico, sino que "[...] esto refleja problemas en el diseño, organización, levantamiento y procesamiento de algunos censos [...]" (1994: 24). No obstante, al analizar de manera comparada todos los recuentos censales pueden observarse dos tendencias naturales en la población indígena. Por un lado, su disminución en proporción al total nacional — cuadros 1 y 2 - , que se explica al tomar en consideración que la población indígena va paulatinamente descendiendo debido sobre todo a la aculturación y al mestizaje. Y, por otra parte, el aumento del número total de indígenas — cuadro 3-, por lo que la desaparición inevitable de esta población, que muchos intelectuales y gobernantes han pronosticado desde la Independencia hasta la década de 1970, no parece que vaya a producirse.

\section{Cuadro 1: Censos de población, 1895-2000}

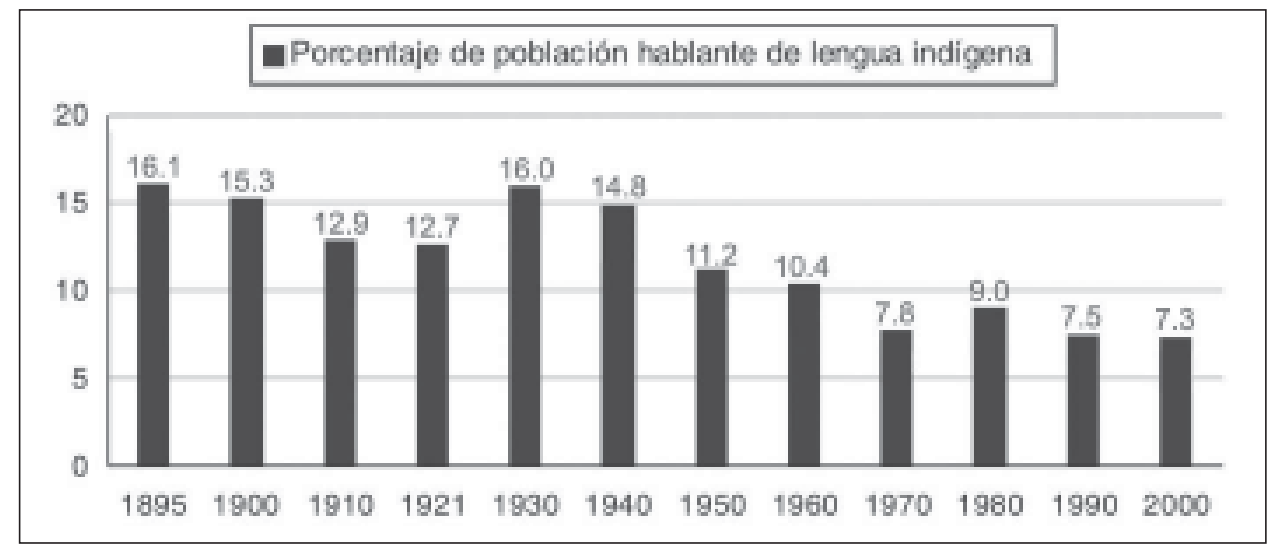

Fuente: INEGI. La población indígena en México. México: Instituto Nacional de Estadística, Geografía e Informática, 2004. 
Cuadro 2: La población hablante de lengua indígena respecto al resto de la población, 1895-2000

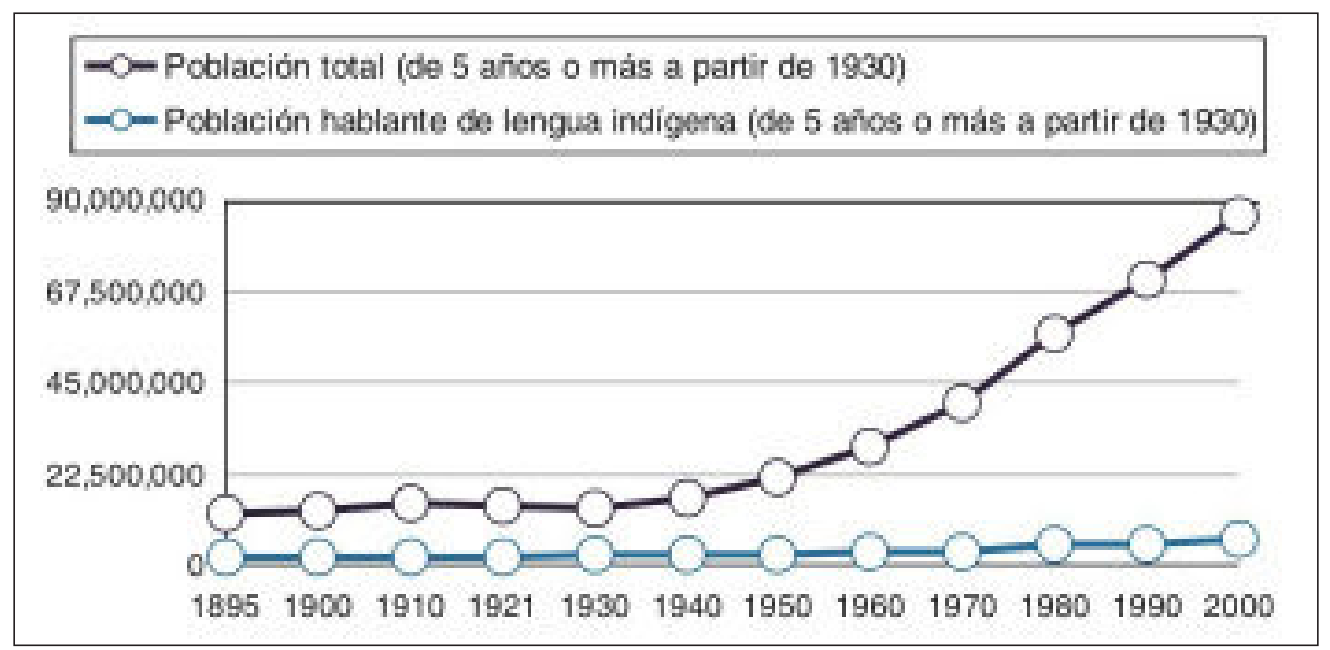

Fuente: INEGI. Censos de población, 1895-2000.

Cuadro 3: Cifras totales de población hablante de lengua indígena en los censos de población, 1895-2000

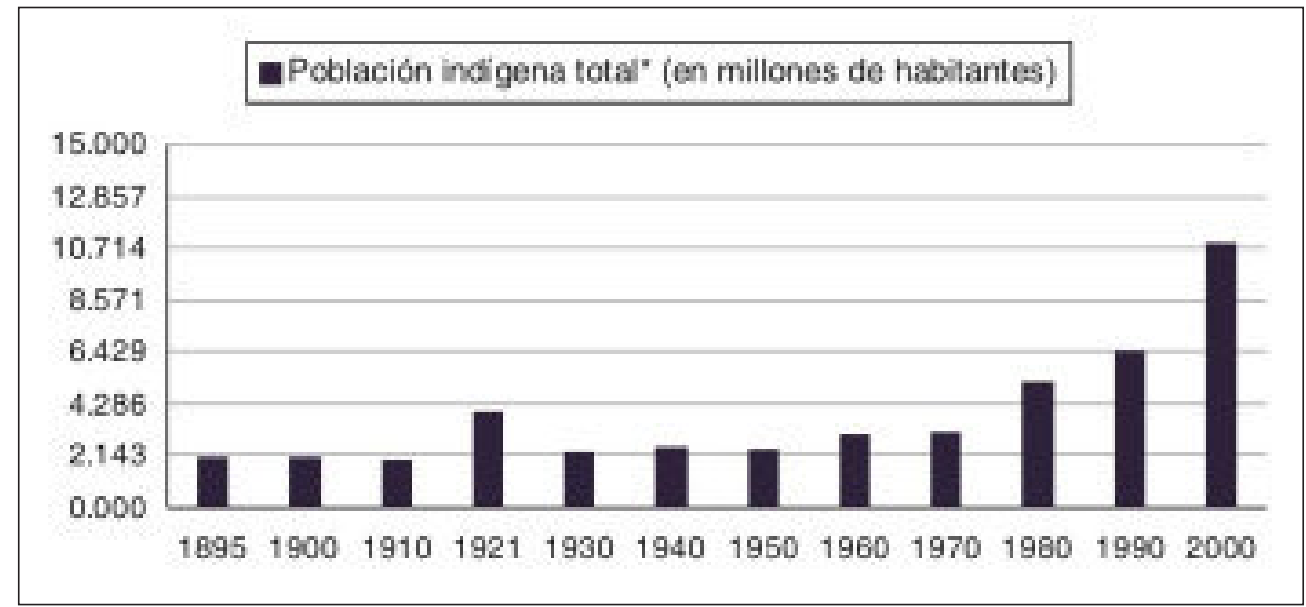

*HLi de 1895 a 1910, de raza indígena en 1921; HLI de 5 años o más de 1930 a 1980; y HLi de 5 años o más combinada con habitantes de hogar, HLI en 1990 y 2000.

Fuente: INEGI. Censos de población, 1895-2000. 
La población indígena mexicana ha estado presente en los censos de población desde el primero de ellos, 1895, hasta el último, 2000. El censo de 1895 es el primero que se basa en la lengua hablada para definir a la población indígena. El hecho de que la administración y los estudiosos califiquen de "modernos" a los censos que emplean la lengua hablada para diferenciar a los indígenas hace prever que este criterio habrá de gozar de gran éxito en el futuro, resistiendo el paso del tiempo y las diferentes perspectivas respecto al tema indígena hasta la actualidad.
Aunque puede diferenciarse, de manera implícita, entre hablantes de lengua indígena monolingües y bilingües en el primer censo moderno, ambos grupos se distinguen de manera explícita y sistemática desde 1930 — cuadro 4 - apareciendo también el sexo de los hablantes. Además, se registran los denominados Estados eminentemente indígenas, ${ }^{20}$ que son los que poseen una población hablante de lengua indígena superior a 7.5\% (Manrique, 1994), así como los municipios mayoritariamente indígenas, los que cuentan con un porcentaje de $40 \%$ o más HLI.

\section{Cuadro 4: Porcentaje de población HLI monolingüe y bilingüe en los censos de población, 1930-2000}

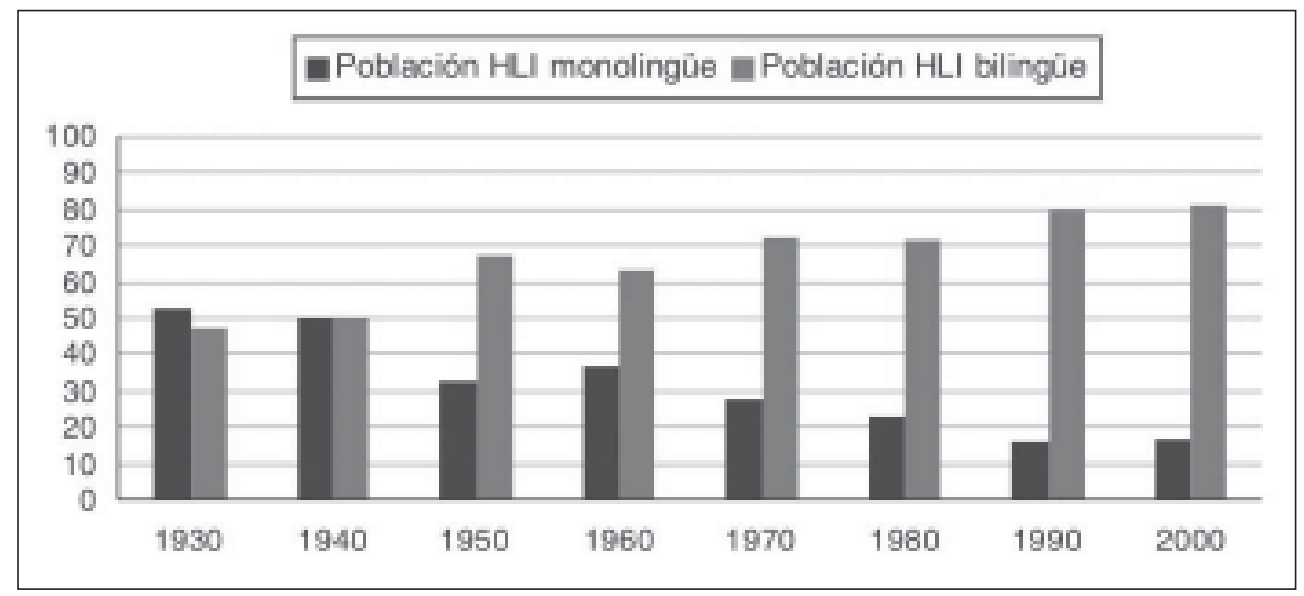

Fuente: INEGI. Censos de población, 1895-2000.

El número de lenguas registradas en cada censo es muy variable. En los primeros las cifras son muy elevadas: 52 en 1895, 50 en 1910, 43 en 1921, y no demasiado fiables, puesto que aparecen en ellos lenguas que no vuelven a figurar después ${ }^{21}$ (Manrique, 1994: 8). En 1930 aparecen censadas 36 lenguas; en 1940 la cifra disminuye a 33; continúan descendiendo a 29 para 1950; en 1960 se registran 30; 1970, 31; y en 1980 el total de lenguas indígenas registradas es de 40; comenzando un ascenso que culminará en 1990 con
92; finalizando con las 85 captadas en el censo de 2000 (Valdés y Menéndez, 1987: 11).

Puede observarse que de unas décadas a otras se producen abruptos altibajos, que no corresponden a la desaparición de algunas lenguas ni al nacimiento de otras — no podría ser así puesto que todas son prehispánicas- Las lenguas minoritarias se incluyen en el rubro "otras lenguas" (Valdés, 2003). Podría afirmarse que el número de lenguas que aparecen nombradas da cuenta sobre el interés que tiene el Estado 
en cada momento por obtener información acerca de la población indígena.

En los tres primeros censos modernos, 1895, 1900, 1910, la población se divide en tres grandes grupos: "los que hablan o no español", "los que hablan o no lenguas indígenas" y "los que hablan o no idiomas extranjeros". Es posible que esta opción de adscripción a idiomas no mexicanos, que no volverá a repetirse en el futuro, esté relacionada con las facilidades que durante el Porfiriato se ofrecen a las ideas e inversiones extranjeras, sinónimos de desarrollo y modernización. En esta temprana época de formación del proyecto nacional, probablemente se consideraba beneficiosa la intervención extranjera en dicho proyecto.

El primer censo arroja una cifra de 2.04 millones de indígenas, que constituye $16.01 \%$ de un total de población de 12.70 millones de habitantes. A pesar de que se registran 52 lenguas indígenas, la gran mayoría de la población HLi habla una de las cinco mayoritarias: náhuatl, maya, lenguas zapotecas, otomí y lenguas mixtecas; que siguen siendo, junto con el tzotzil, las más habladas en la actualidad.

A pesar de que la ideología liberalista imperante en México del siglo xIx tiene como máxima la igualdad entre todos los ciudadanos, se refleja en los censos la diferencia indígena, ${ }^{22}$ lo que aparentemente supone una contradicción. Probablemente figura este dato porque resulta ineludible registrar a un grupo tan numeroso, pero puede interpretarse que se trata de una información que posee carácter coyuntural, constituyendo una realidad momentánea, puesto que la tendencia del momento es la asimilación y la previsión de futuro, con bastante seguridad esta información desaparecerá del censo.

El censo de 1900 informa que habitan 2.07 millones de indígenas en México, cifra que representa 15.27\% de un total de población de 13.60 millones. En el censo levantado en 1910 se manifiesta que la población india mexicana asciende en este año a 1.96 millones, que constituye $12.93 \%$ de un total de 15.16 millones de habitantes.

La Revolución mexicana supuso un replanteamiento de las ideas sobre los indígenas, que se materializó en las obras de los antropólogos indigenistas posteriores (De la Peña, en INı, 2000). También se reflejan los nuevos planteamientos en los censos de población 1921, 1930, 1940, 1950 y 1960, que permiten observar el interés, característico del "periodo indigenista", por conocer en profundidad el "problema indígena". Así, en 1921 la lengua indígena aparece combinada con el criterio racial y en los censos 1940 y 1950 se realizan sendos ensayos de aplicación de criterios culturales.

En el iv censo se ofrece una cifra de hablantes de lengua indígena de 1.82 millones de personas, que supone $12.70 \%$ de un total de 14.33 millones de habitantes. No obstante, las cifras oficiales indican que la población indígena representa $28.90 \%$ del total nacional porque se añade a la cuestión sobre lengua hablada una pregunta acerca de autoadscripción racial..$^{23}$

Este censo refleja la preocupación del momento, tras la coyuntura revolucionaria, en la que tanta importancia han tenido los indígenas, de recoger fielmente a dicho grupo. El resultado del altísimo porcentaje en absoluto perjudica la creación del proyecto nacional, que pretende categorizar, sin homogeneizar de momento a la inmensa mayoría como "pueblo", concepto que los indígenas engruesan de manera relevante.

La Constitución de 1917 establece que toda la población tiene los mismos derechos, los mismos deberes y es igual ante la ley, independientemente de su origen o su color de piel. A partir de 1930 se materializa el mandato constitucional de no discriminación en los censos de población y deja de utilizarse definitivamente el criterio de raza en ellos, al igual que desaparece de todo el resto de documentación oficial.

El quinto censo, 1930, se dedica de manera exhaustiva a la descripción de las poblaciones indígenas, 
pero ésta se limita al ámbito lingüístico, ${ }^{24}$ se introduce la innovación de incluir series estadísticas en el ámbito nacional y estatal, y se divide a los hablantes de lenguas indígenas entre bilingües y monolingües, ${ }^{25}$ distinguiendo su sexo. También en dicho censo se restringe por vez primera la población hablante de lengua indígena a individuos de cinco años o más, puesto que se considera que es a esa edad cuando las personas han adquirido plenamente la habilidad lingüística.

Se manifiesta en los resultados del conteo que 2.25 millones de habitantes, de un total ${ }^{26}$ de 14.04 millones, hablan alguna lengua indígena: 1.18 millones de manera exclusiva y 1.06 millones hablan también español, lo que supone $16.02 \%$ de hablantes de lengua indígena respecto al total de población nacional.

La mencionada introducción de gran cantidad de información referida a hablantes de lengua indígena en este censo se debe, en opinión de M. A. Bartolomé (INI, 2000), al interés gubernamental por medir los efectos de las campañas educativas propiciadas por él. La "educación del pueblo" que el Estado nacido de la Revolución ha puesto en marcha comienza a dar resultado, como puede apreciarse a partir del censo de 1940, con la disminución de los monolingües. Dicha educación es la materialización del empeño de las políticas indigenistas por el conocimiento de la problemática situación de los indígenas actuales, con el fin de mejorarla para facilitar su integración al proyecto nacional, conservando sus características positivas - las que concuerdan con el papel que juega su estereotipo en la idea de nación-y abandonando las negativas que los relegan, y con ellos al resto de la nación, al subdesarrollo.

En el censo de 1940, los indígenas ascienden a 2.49 millones de habitantes, de los cuales 1.25 millones son bilingües y 1.23 millones monolingües. La población india representa $14.83 \%$ del total nacional, 16.78 millones de personas. En el VII censo, levantado en 1950, se establece que 2.4 millones de habitantes, $11.24 \%$ de una población de 21.77 millones, son hablantes de alguna lengua indígena. El monolingüismo continúa descendiendo en este VII censo, contabilizándose 795,069 monolingües frente a 1.65 millones de bilingües.

Los censos de 1940 y 1950 constituyen excepciones en lo que refiere a los criterios de clasificación, puesto que, al igual que el de 1921, combinan diversos criterios. Utilizan el lingüístico, como el resto de los censos, pero, siguiendo las teorías de la antropología indigenista mexicana del momento, se llevan a cabo ensayos de empleo de criterios de carácter cultural. Aparte de preguntarse por la lengua hablada, se pregunta por el vestuario y la alimentación, ya que los trajes locales y la dieta basada en el maíz se consideran rasgos indígenas.

Los estudiosos consultados, a pesar de que califican estos censos de notorio fracaso debido a que en ambos el número de personas que se identificó como indígena constituía prácticamente la mitad de la población mexicana, afirman que consiguieron el objetivo de avanzar en la implantación de la ideología del mestizaje: "La combinación estadística de los dos criterios, el lingüístico y el cultural [...], aunque fuera limitada, 'habría de servir para determinar el curso de la transformación del indio en mestizo" “ (Valdés y Menéndez, 1987: 9). El criterio de identificación cultural de los indígenas deja ver que la frontera entre indígenas y no indígenas en México no está claramente delimitada; atendiendo a criterios culturales, los indios y el resto de la población se confunden.

A causa del resultado de los ensayos realizados en los recuentos anteriores, a partir del VIII censo, realizado en 1960, se decide utilizar exclusivamente el criterio lingüístico. En este conteo de población se estima que la población hablante de lengua indígena supone $10.39 \%$ de un total nacional de 29.14 millones de habitantes, es decir, 3.03 millones de personas, de las cuales 1.92 millones son bilingües y 1.10 millones monolingües. 
En 1970, intelectuales que escriben publicaciones aledañas al censo de población son de la opinión, generalizada hasta la década de 1980, de que la cultura indígena desaparecería de manera gradual, pero irremisiblemente: "Una consecuencia de la naturaleza del proceso histórico de nuestro país, de la forma en que se han dado la irrupción del capitalismo y el desarrollo de su dependencia, de la manera en que los procesos superestructurales han llevado a la formación de una sola nacionalidad, a la necesidad de estimular la 'modernización', así como de la forma en que se ejerce el control político, ha sido la tendencia manifiesta hacia la desaparición de la cultura y de las lenguas indígenas [...]" (Olivera, Ortiz y Valverde, 1982: 10).

Las cuestiones culturales se subordinan a las políticas y se considera inevitable, sin entrar en juicios de valor, la desaparición de la cultura india debido a la imparable modernización. Pero se trata de una corriente ideológica coyuntural, de transición entre el "proyecto indigenista" y el "proyecto pluralista" posterior; pronto, lo étnico resurgirá con una fuerza nunca vista hasta entonces.

Las cifras que el Ix censo, 1970, arrojan 3.11 millones de hablantes de lengua indígena, lo que representa $7.76 \%$ de una población total nacional de 48.22 millones de habitantes. El total de indígenas mencionado se divide en 2.25 millones de bilingües y 859.854 monolingües.

En el x censo, levantado en 1980, se afirma que el número total de hablantes de lengua indígena asciende a 5.18 millones, de los cuales 1.17 millones sólo hablan una lengua indígena y 3.70 millones además hablan español. La población india representa, así, 9.04\% de un total nacional de 57.30 millones de personas.

Instituciones oficiales afirman que, a partir de la década de 1980, resurge la necesidad de disponer de cifras fiables ${ }^{27}$ sobre el tamaño de la población indígena (www.cdi.gob.mx). Cabe preguntarse por qué surge esta necesidad precisamente en esta fecha, se puede afirmar que la respuesta es el ya citado auge de la valoración de la pertenencia étnica, que se produce gracias a la situación nacional e internacional, propiciatoria de este fenómeno. Organismos internacionales, como Naciones Unidas, expresan la necesidad de conocer en toda su complejidad la situación indígena. Los motivos que se aducen son de dos tipos; por un lado, para conseguir el respeto y la supervivencia de la etnicidad; y, por otro, “[...] es importante llevar a cabo un análisis más detallado de las dimensiones que abarcan la pobreza y la marginación, así como de otros indicadores que permitan identificar la profundidad y complejidad de los rezagos que prevalecen entre la población indígena" (www.un.org).

El criterio del censo de 1980 es únicamente el lingüístico, pero aún así se produce un aumento inexplicado de hablantes de lengua indígena respecto al censo anterior. No existe ninguna evidencia que indique que en este decenio esta población haya crecido de manera desproporcionada, por lo que deben considerarse motivos externos a los demográficos.

L. M. Valdés opina que se debe a la subnumeración previa, tanto del censo de 1970 como de todos los anteriores. No es coincidencia que en la década de 1980 nacieran las políticas de la identidad, es decir, que comenzara la valoración de la etnicidad. La autora afirma: "La formación de asociaciones y organizaciones indígenas, hasta la creación de centros coordinadores indigenistas y otras instancias administrativas, han fortalecido la presencia de los indios mexicanos en los censos" (Valdés, 1989: 41). Ciertamente es así, pero se debe ir más allá e intentar establecer la causa por la que proliferan en el decenio 1970-1980 los organismos mencionados. La coyuntura nacional e internacional permite, e incluso provoca, la aparición de dichas instancias, que, a su vez, influyen en que el número de indígenas censado aumente. Es decir, la valoración de la diferencia, a nivel internacional y, por tanto, nacional, trae consigo que el número oficial de indígenas sea mayor. 
Hay que añadir que la cifra de población india que establece este $\mathrm{x}$ censo, pese a ser muy superior a la de los anteriores, no cuenta con total aprobación: la Comisión Nacional de Población estima que el número total de indígenas era de diez millones, mientras que las organizaciones indígenas afirman que la cifra total es de doce millones (Valdés, en Muñoz, 1992).

En los censos 1990 y 2000 , en aras del registro fiable de la heterogeneidad característica de la nación mexicana, ahora valorada de manera positiva, se cuestionan incluso los criterios clasificatorios que han permanecido indiscutidos desde 1960. Comienza a resultar importante la población hablante de lengua indígena menor de cinco años, que hasta ese momento había pasado desapercibida en los conteos censales. De este modo, se pone en marcha el recuento de los "hogares HLI". ${ }^{28}$ No se trata de un criterio nuevo, sino de una manera de completar el lingüístico que ha dejado de ser considerado operativo en el momento en que la diferencia ha pasado a ser un valor.

Según el censo general de población y vivienda levantado en 1990, la población HLI de cinco años y más asciende a 5.28 millones de personas, de las cuales 4.23 millones son bilingües y 836.224 monolingües, lo que representa $7.48 \%$ de un total nacional de 70.56 millones de personas. Por su parte, la población menor de cuatro años que habita en hogares HLI es de 1.13 millones de personas. La suma da como resultado una población indígena total de 6.41 millones.

Además, este XI censo ofrece amplia información sobre numerosos aspectos de las poblaciones indígenas, no con el objetivo de la integración, como sucedía durante el anterior "periodo indigenista", sino con el del conocimiento de algo valioso que hasta este momento había sido infravalorado. Los resultados no desilusionan, se cuentan 92 lenguas indígenas. Este asombroso aumento — de 40 en 1980 a 92 en 1990_ se debe en parte a la fuerte emigración de indígenas mayas a México, procedentes de Guatemala. No obstante, es imprescindible que se considere necesario tener en cuenta esta especificidad para que aparezca reflejada.

El criterio lingüístico continúa vigente en el censo 2000, con la modificación de incluir en el recuento la población menor de 5 años. Pero deja de ser criterio único, al combinarse con el autoadscriptivo. ${ }^{29} \mathrm{Sin}$ embargo, no se interroga a toda la población sobre su pertenencia étnica, sólo a una muestra formada por $10 \%$ del total nacional. Las preguntas ${ }^{30}$ realizadas responden a la pretensión de llevar a cabo un ensayo con el fin de decidir si se acepta o no el criterio para los censos posteriores.

Se deriva de la muestra censal que 5.26 millones de mexicanos se autoadscriben a alguna etnia indígena, a pesar de que 1.10 millones de ellos no hablan ninguna lengua amerindia, y que 1.95 millones de habitantes no se consideran indígenas aunque sí hablen lenguas prehispánicas. Esta discordancia de cifras puede achacarse a que tanto el término "indígena" o "indio" como el concepto "pertenencia" causan incomodidad a los encuestados (www.cdi.gob.mx).

Las cifras sobre población indígena del XII censo de población, 2000, indican que habitan en México 6.32 millones de hablantes de lengua indígena, de los cuales 1.07 millones son monolingües y 5.12 millones son bilingües, lo que supone $7.35 \%$ del total nacional, que asciende a 85.93 millones de habitantes. Por otra parte, 10.91 millones de personas pueden ser consideradas indígenas debido a que residen en hogares HLI.

Como viene siendo habitual desde la década de 1980, numerosos autores e instituciones oficiales no dan por válidas las cifras que el censo arroja, por considerarlas subnumeradoras. El Consejo Nacional de Población estima que la cifra total de población indígena, con base en las respuestas sobre lengua hablada, residencia en hogares HLI y pertenencia étnica, es de 12.7 millones en 2000 (www.conapo.gob.mx), dicha cifra la respalda también la Comisión Nacional 
para el Desarrollo de los Pueblos Indígenas, apoyada por Naciones Unidas (www.un.org). La discusión en torno a las cifras se nutre con nuevos participantes, instituciones que afirman que el número de indígenas es bastante superior al que refleja el censo. Las cifras disminuyen, pero obtienen difusión voces que no las dan por válidas.

\section{Conclusiones}

En este artículo se recogen los criterios utilizados para decidir qué población es étnicamente diferenciada y cuál no en los doce censos modernos de población, así como los resultados numéricos referidos a estas poblaciones que en ellos aparecen. Los datos escogidos para el estudio son los mencionados porque se entiende que el Estado, a través de los recuentos de población, emite un discurso en el que define a los indígenas. Esta definición no es estática, sino que va cambiando en función del momento histórico, como causa y, simultáneamente, consecuencia de la variación de los modos de realizar los conteos y las cifras que éstos arrojan. Utilizando la mencionada información se relacionan los criterios y los resultados numéricos con las corrientes ideológicas y políticas vigentes en cada momento. Con la definición de población indígena que el Estado confiere a través de los censos, se otorga parte de su identidad a los indígenas. De este modo, el Estado participa en el proceso de construcción de identidades étnicas con el objetivo de que formen parte del proyecto nacional vigente en cada periodo, lo que constituye la autoimagen que el Estado mexicano ha querido proyectar en cada momento histórico a lo largo de su vida independiente.

El Estado mexicano ha diferenciado, desde su nacimiento, a los indígenas. Para llevar a cabo esta distinción el primer paso consiste en definir qué entiende el Estado por "indígena". Puesto que la identidad es negativa, a partir de la necesidad no tanto de diferenciar a los indígenas como de diferenciarse de ellos, la nación se está definiendo a sí misma. Así, la identidad nacional mexicana se define, en gran medida, otorgando identidad a los indios. La identidad étnica se construye en un proceso de negociación en el cual el Estado participa de modo relevante, a partir del establecimiento de fronteras basadas en rasgos diferenciatorios seleccionados y estereotipados, y por tanto subjetivos, que, a su vez, conforman los criterios de clasificación —de los que sólo necesitan "los otros"; los grupos dominantes no, porque constituyen la norma-. Los criterios clasificatorios se modifican con el tiempo para adaptarse a las necesidades de los grupos hegemónicos y a las distintas circunstancias históricas, de esta manera demuestra que la identidad es dinámica y plástica. La asignación de identidades étnicas no es arbitraria, sino que responde al objetivo de la élite política de formar, en torno a sí, el proyecto de nación.

Hasta el día de hoy no existe una definición unánimemente aceptada de "indígena". Sin embargo, a lo largo de la historia del México independiente se ha definido a los indígenas de diversas maneras, plasmadas en los criterios clasificatorios utilizados en los censos de población, lo que ofrece abundante información acerca de la nación que en cada momento se proyecta. Dependiendo del criterio clasificatorio escogido, los resultados numéricos de los censos varían, apareciendo reflejados altibajos que puede inferirse constituyen un reflejo del momento histórico-político, de la ideología del momento, que modela, a través del empleo de unos u otros criterios, una población indígena más o menos relevante.

Los censos mexicanos modernos han permanecido fieles al criterio lingüístico desde el principio. A pesar de que la utilización de un criterio único normalmente da como resultado un número poco elevado de indígenas; esa ha sido la norma, con notables excepciones, en México. Tal vez podría afirmarse que el uso generalizado del criterio único lingüístico, que 
arroja por lo general cifras bastante moderadas, está relacionado con la tendencia asimilacionista e integracionista que el Estado ha manifestado hasta hace pocas décadas respecto a los indígenas contemporáneos. Esta aseveración se refuerza si se tiene en consideración que el Estado acepta las cifras presuntamente subnumeradas, y no acata las supuestamente sobrenumeradas.

Durante el "periodo liberal" prerrevolucionario se proyecta una nación homogénea y moderna que pretende imponerse a través de la asimilación de la población indígena y su conversión a la igualitaria categoría de "ciudadanos". Probablemente, el hecho de que aparezcan diferenciados los indígenas en los censos I, II y III se deba a que al Estado le es imposible ignorar la discriminación de que es objeto esta población como herencia de la época colonial, lo que no deja posibilidad de obviar el registro de estos grupos tan diferenciados.

Durante el indigenismo, el objetivo es integrar a los indígenas para lograr una nación desarrollada, pero sin negar la herencia prehispánica, por lo que se les identifica como poblaciones sumamente problemáticas a las que hay que insertar, abandonando sus rasgos "negativos" y conservando los "positivos", en el desarrollo nacional. Los seis censos que se levantan entre 1921 y 1970 son sumamente irregulares. Destacan por las altas cifras que arrojan respecto a población indígena: el IV cerca de 30\%; el VI y VII entre 40 y 45\%, aunque estas últimas se considerarán erróneas, por lo que apenas obtendrán difusión, dándose por válidas las cifras que únicamente se refieren a población HLi, inferiores ambas a 15\%. En los censos V y VIII aparecen cifras moderadas debido a lo sucedido en los que les precedieron. En cuanto al IX, la cifra de HLI que en él aparece es excepcionalmente baja, lo que podría deberse, entre otras cosas, a que las corrientes políticas imperantes en el momento, de carácter marxista, no dan demasiada importancia a lo étnico, sino que lo subordinan al concepto de clase social.

Alrededor de 1980 el proyecto nacional comienza a variar, empezando el Estado a autopercibirse como "pluralista", por lo que se define a los indígenas como poblaciones con un gran valor cultural que hay que conservar. El x censo arroja, en consecuencia, una cifra comparativamente muy alta, $9 \%$ frente a $7.76 \%$ del IX y $7.48 \%$ del xi. En el XI censo se cuestiona el criterio lingüístico y se intenta perfeccionar con el conteo de hogares HLI, el resultado es que se cuentan 6.36 millones de indígenas. En el XII aumenta el número de hablantes de lengua indígena respecto al anterior, la cifra no es muy destacable por sí sola, pero si se combina con el recuento de hogares HLI, se obtiene una cifra de 10.91 millones de indígenas, que, además, es discutida por numerosos organismos, nacionales e internacionales, pues insisten en que la población indígena mexicana asciende a 12 millones de personas. Además, se prueba con un criterio nuevo, realizándose un ensayo de autoadscripción, que, aun sin tener mucho éxito, deja ver el interés del Estado por conocer lo que los habitantes piensan respecto a su pertenencia étnica. Muy destacable en este periodo es el número de lenguas indígenas registradas, puesto que indican el interés del Estado por recoger y respaldar la diferencia. Frente a la regularidad del periodo anterior — exceptuando las 43 del IV, entre 29 y 36 lenguas en los censos V, VI, VII, VIII y IX-, en el x se contabilizan 40, que ascienden sorprendentemente a 92 en el XI censo, quedando reducidas a 85 en el XII, cifra que, aunque inferior a la anterior, no deja de ser muy elevada.

Concluyendo, podría afirmarse que los cambios en los censos de población descritos obedecen, en gran medida, a modificaciones en las necesidades de autopercepción del Estado: en los siglos XIX y Xx las aportaciones que los indígenas contemporáneos pueden hacer a la construcción del proyecto nacional son escasas, por lo que su conversión en ciudadanos primero, y en "pueblo" después, es conveniente; por el contrario, desde hace algunas décadas se considera que los indígenas actuales sí tienen mucho que aportar al proyecto de nación, por lo que se valora su crecimiento. 


\section{Notas}

${ }^{1}$ Este artículo forma parte de una investigación que la autora está realizando bajo la dirección del Dr. Pedro Pitarch Ramón, para el programa de Doctorado en América Latina Contemporánea del Instituto Universitario de Investigación Ortega y Gasset.

${ }^{2}$ Puede afirmarse que los grupos dominantes permiten que los grupos subalternos escojan determinadas identidades; no cualquier adscripción identitaria es posible en cualquier momento. La identidad se construye a través de la negociación entre el Estado y los grupos indígenas, resultando un conjunto de rasgos definitorios, de los cuales algunos son estereotipos impuestos desde fuera y otros rasgos son enfatizados desde el interior de los grupos étnicos, pero para la asignación de esta identidad construida es necesaria la conformidad de los grupos dominantes.

${ }^{3}$ Concepto creado por C. Lévi-Strauss, citado por J.M. Benoist, 1981, en La identidad: seminario interdisciplinario dirigido por Claude Lévi-Strauss. 1974-1975. La “destotalización” del propio yo es el primer paso en la construcción de la identidad; el "destotalizador" primario es el nombre propio, puesto que implica que se necesita de un término que diferencie al individuo del resto.

${ }^{4}$ E. Malvido y M.A. Cuenyas, en su libro Demografía histórica de México, siglos XVI-XIX, utilizan, para responder a esta cuestión, la obra de W. Kula, 1980, Las medidas y los hombres, Siglo xxi, México.

${ }^{5}$ Se han utilizado los trabajos sobre censos de población de L. M. Valdés (1987, 1989 y 2003), D. Yanow (Greenwood y Greenhouse, 1998) y Roosens (Pujadas, 1993).

${ }^{6}$ En el caso concreto que nos ocupa, la población indígena en los censos, así lo afirman L. M.Valdés y M. T. Menéndez: "La forma como son tratados los datos en cada censo, la amplitud o restricción de las cifras que presentan y las explicaciones de los encargados de realizar los censos, en las introducciones, nos demuestran, de alguna manera, el espacio que los grupos indígenas han ocupado en el plano nacional; en tanto los censos constituyen la fuente principal de información sobre la población de un país" (Valdés y Menéndez, 1987: 7).

7 "Negro" es la categorización fundamental en Estados Unidos. Constituye una categoría incluyente por la regla de one-droprule, "sólo una gota basta", es decir, cualquier persona que posea un antepasado de raza negra es considerada "negra", es una identificación que no tiene ningún componente autoadscriptivo ni cultural y es, además, determinante para la vida del individuo, más parece un estigma que una categorización. Con "indio" sucede lo contrario, funciona como categoría excluyente; en Estados Unidos hay que probar que se poseen antepasados indígenas para poder ser categorizado como tal, y en esta identificación sí son importantes las tradiciones. Es frecuente, en países donde el objetivo es el mestizaje, que los indígenas dejen de serlo por dejar de hablar su lengua materna o por perder las tradiciones de sus ancestros. Estados Unidos trata de excluir a las personas de raza negra, por eso les estigmatiza con una identificación de la que no pueden escapar; por el contrario, tanto Estados Unidos como muchos otros países americanos buscan integrar a los indígenas, por eso les facilitan el abandono de su identidad.

${ }^{8}$ De hecho, ha dejado de utilizarse en la mayoría de países con la notable excepción de Estados Unidos. En Europa y América Latina, el término "raza" ha evolucionado para acabar convirtiéndose en "etnia"; sus significados son diferentes, puesto que la primera se ocupa del ámbito biológico y la segunda del cultural, pero frecuentemente se utilizan como sinónimos; no obstante, esta evolución de denominaciones es reflejo de distintos momentos históricos.

9 J. J. Pujadas, en Etnicidad. Identidad cultural de los pueblos , describe un estudio, llevado a cabo por Roosens, sobre población indígena en los censos de población de Canadá. Roosens trata los intereses políticos que se esconden tras las categorías censales. En Canadá, el criterio utilizado para definir quién es indígena es la filiación. Según este criterio, para que alguien pueda inscribirse en el censo como indígena tiene que demostrar que posee "sangre indígena", además es obligatoria la "pureza de sangre", es decir, ser descendiente de dos padres indígenas. El autor afirma que "es evidente que la actitud de las autoridades es la de ralentizar los procedimientos de adquisición del estatuto indígena como medio para intentar restar fuerza al proceso creciente de revitalización de la identidad y de los movimientos étnicos canadienses" (Pujadas, 1993: 19). Las autoridades canadienses ofrecen multitud de ayudas a las poblaciones indígenas, como compensación del expolio de tierras del que fueron víctimas en el pasado. Es necesario que el Estado controle el censo de población para evitar la "multiplicación" de la población indígena dispuesta a ser objeto de ayudas estatales. Por otro lado, la población india canadiense está de acuerdo con el férreo control de los censos, puesto que el respeto y las ayudas de los que se benefician disminuirían si su número creciera desmedidamente. La "Asociación de Mestizos e indios sin Estatus" lucha por el 
reconocimiento de los derechos de los mestizos y de los indios que no son identificados como tales por el Estado canadiense. Los mestizo

s pueden o no ser reconocidos como indios; según las leyes canadienses, es indio el hijo de padre indígena, pero no el de madre indígena, basándose en la filiación tradicional étnica, de carácter patrilineal. El Estado, a través de las ayudas minoritarias, logra que el grupo de población legalmente indígena no crezca, por su parte, la población legalmente indígena no reclama los derechos de los indígenas sin estatus, porque temen perder o ver disminuidas las ayudas que el Estado les otorga, a pesar de que si la población indígena aumentara numéricamente tendría mucha más fuerza para negociar con el Estado.

${ }^{10}$ Según una clasificación que A.Peyser y J. Chackiel (CELADE, 1994) extraen de publicaciones de cepal, existen tres grupos de países en América Latina en función del peso relativo de las etnias en la población nacional:

1. Países cuya población indígena tiene un peso muy significativo a nivel nacional. Se encontrarían en este grupo México, Perú, Bolivia, Ecuador y Guatemala.

2. Países cuyo número total de indígenas es relevante, aunque menos que en los del primer grupo. Este grupo estaría formado por Colombia, Chile, Honduras, Panamá y Paraguay.

3. Países cuyo total de población indígena es menor. Integrarían este grupo Brasil, Venezuela y Colombia (que pertenece a ambos grupos).

Es en el tercer grupo de países donde puede aplicarse, y de hecho se aplica, el criterio de ubicación geográfica, puesto que sus poblaciones indígenas comparten el rasgo distintivo de habitar en ámbitos de selva.

${ }^{11}$ Tradicionalmente ser indio ha sido, en gran medida, un estigma, por ello, muchas personas que serían indígenas según otros criterios no se autoadscriben como tales. También es evidente que a partir de la valoración de la etnicidad, en auge desde la década de 1980, ser indígena puede representar muchas ventajas y a causa de ello pueden identificarse como indígenas personas que según otros criterios no pertenecen a este grupo de población.

${ }^{12}$ Según A. Caso: "Es indio el que se siente pertenecer a una comunidad indígena y es una comunidad indígena aquella en que predominan elementos somáticos no europeos; que habla preferentemente una lengua indígena; que posee una cultura material y espiritual en donde hay elementos indígenas en fuerte proporción y que, por último, tiene un sentido social de comunidad aislada dentro de las otras comunidades que la rodean, que la hace distinguirse a la vez de los pueblos blancos y de los mestizos". América Indígena, Vol. III, I.I.I., México, 1948 (Valdés y Menéndez, 1987: 10).

${ }^{13} \mathrm{M}$. Gamio, en Consideraciones sobre el problema indígena, ofrece una clasificación basada en las características culturales: “a) propiamente indígenas, o sea de origen prehispánico, y además aquellas muy contadas que se crearon o inventaron después de la conquista sin influencias de culturas extranjeras;

b) de cultura extranjera que son principalmente las de origen europeo;

c) mixtas, o sea aquellas que resultan de la mezcla de las dos culturas anteriores"(Valdés y Menéndez, 1987: 10).

${ }^{14}$ Además, no existe consenso en afirmar que con el cambio cultural se pierde la etnicidad; G. Bonfil (INI, 2000) afirma que el cambio cultural fortifica la identidad cuando el grupo controla dicho cambio.

${ }^{15}$ Aunque las dos objeciones expuestas sean en apariencia contradictorias pueden resumirse en una sola: "población hablante de lengua indígena" y "población indígena" son dos conceptos que no se corresponden el uno con el otro.

${ }^{16}$ Así lo expresa L.M. Valdés: "Los censos de población están elaborados para informar sobre la lengua como un concepto, y no sobre la población indígena que es quien la habla y menos aún sobre las características de esta población. Así, podemos afirmar que los censos de población no informan sobre la población indígena como grupo diferenciado del resto de la población, sino sobre la distribución de las lenguas autóctonas que aún privan en México" (Valdés, 1989: 30).

${ }^{17}$ Un ejemplo de las diferentes cifras que pueden arrojar los censos en función del criterio que se escoja son los resultados de las estructuras por edades de la población indígena, si se utiliza para recoger los datos del criterio lingüístico, suele suceder que los hablantes de lenguas indígenas son mayoritariamente personas de edad avanzada, mientras que si el criterio elegido es la autoasignación, debido al éxito del que goza entre la juventud el resurgir étnico, las cifras arrojan una edad media de los indígenas mucho menor.

${ }^{18}$ Las técnicas empleadas en el conteo pueden influir en los resultados censales. Si se ponen abundantes medios a disposición de la oficina del censo para que se realice el conteo, significa que existe un interés en que la cifra resultante sea elevada, si, por el contrario, se realiza con pocos medios, 
significa que no es prioritario para el gobierno destacar la heterogénea población que compone la nación.

${ }^{19}$ Definido como: "[...] fenómeno mediante el cual los indios desaparecen de las estadísticas nacionales [...] el problema no es casual, sino que está enmarcado en las políticas de asimilación que el Estado diseña para incorporar a los indios mexicanos a la 'sociedad nacional'" (Valdés, 1989: 39).

${ }^{20}$ Los estados con mayor proporción de población HLI respecto al total estatal, en todos los censos modernos, aunque el orden varía, son Yucatán, Oaxaca, Campeche, Puebla, Veracruz, Hidalgo, Chiapas y Quintana Roo (mayoritario sólo a partir de 1930). Los estados con mayor número absoluto de hablantes de lengua indígena en los doce censos, también variando su orden de un recuento a otro, son: Oaxaca (que permanece siempre en primer lugar), Puebla, Yucatán, Veracruz, Hidalgo y Chiapas.

${ }^{21}$ Parece que se trata de sinónimos con los que ha habido problemas de traducción.

${ }^{22}$ No sólo aparecen reflejados los hablantes de lengua indígena, además, aparece registrado un elevado número de lenguas.

Puede explicarse esta cifra atribuyéndola a malas transcripciones y mal entendimiento entre encuestadores y población indígena, debidos a que en esta temprana época los métodos de recuento son rudimentarios.

${ }^{23}$ Se pregunta lo siguiente: "A qué raza se siente pertenecer: A) raza blanca, B) mestiza, C) india” (Valdés, 1989: 31-32).

${ }^{24} \mathrm{El}$ retorno al criterio único basado en la lengua hablada no entra en contradicción con la ideología imperante en estos años, como podría parecer a primera vista, puesto que las lenguas indígenas son indiscutiblemente herencia prehispánica y la valoración de ésta es característica del "periodo indigenista". ${ }^{25}$ El censo de 1930 es el primero en que aparecen las denominaciones "monolingüe" y "bilingüe", pero esta información se registra ya en 1895, implícita en la combinación de dos de los grupos incluidos en el I censo, "los que hablan o no español" y "los que hablan o no alguna lengua indígena". ${ }^{26}$ A partir de este $\mathrm{V}$ censo se contabiliza únicamente a la población hablante de lengua indígena mayor de 5 años, por ello, en las cifras que en este escrito aparecen sobre población total nacional entre 1930 y 1990 únicamente se incluye la población nacional de 5 años o más.

${ }^{27}$ En alusión al sesgo político que caracterizó al censo de 1970.

${ }^{28}$ Definidos como aquellos en los que el jefe de familia, su cónyuge o algún otro miembro, exceptuando los empleados domésticos, es hablante de lengua indígena.
${ }^{29}$ Como ha podido observarse, México suele ser cauteloso a la hora de innovar criterios clasificatorios, y no le faltan motivos, teniendo en cuenta los resultados del empleo de criterios culturales, por ello se considera conveniente primero realizar un simulacro para posteriormente decidir si el nuevo criterio se implanta definitivamente.

${ }^{30}$ La batería de preguntas que se ensayó fue:

- ¿Es (nombre) indígena?

- ¿(Nombre) pertenece a un pueblo indígena?

- ¿(Nombre) pertenece a un grupo indígena?

(www.cdi.gob.mx).

\section{Bibliografía}

Anderson, B., 1983, Comunidades imaginadas. Reflexiones sobre el origen y la difusión del nacionalismo, Fondo de Cultura Económica, México.

Bartolomé, M. A., 1997, Gente de costumbre y gente de razón: las identidades étnicas en México, Instituto Nacional de Antropología e Historia, México.

Benoist, J. M. (et al.), 1981, La identidad: seminario interdisciplinario dirigido por Claude Lévi-Strauss. 19741975, Petrel, Barcelona.

CELADE, 1994, Estudios sociodemográficos de pueblos indígenas, Centro Latinoamericano de Demografía, Santiago de Chile.

Chihu, A. (coord.), 2002, Sociología de la identidad, Miguel Ángel Porrúa, México.

Greenwood, D.J. y C.J. Greenhouse (eds.), 1998, Democraciay diferencia. Cultura, poder y representación en los Estados Unidos y en España, Universidad Nacional de Educación a Distancia, Madrid.

Hernando, A., 2002, Arqueología de la identidad, Akal, Madrid. Hobsbawm, E., 1994, "Identidad", Revista Internacional de Filosofía Política, mayo, núm. 3, Universidad Autónoma Metropolitana y Universidad Nacional de Educación a Distancia, Madrid, pp. 5-17.

INEGI, 1993, La población hablante de lengua indígena en México: XI Censo General de Población y Vivienda, 1990, Instituto Nacional de Estadística, Geografía e Informática, México. INEGI, 2004, La población indígena en México, Instituto Nacional de Estadística, Geografía e Informática, México.

INI, 2000, Estado de desarrollo de los pueblos indígenas de México, 2 Volúmenes, Instituto Nacional Indigenista y Programa de Naciones Unidas para el Desarrollo, México. 
Malvido, E. y M. A. Cuenyas (comps.), 1993, Demografía histórica de México: siglos XVI-XIX, Instituto Mora y Universidad Autónoma Metropolitana, México.

Manrique, L., 1994, La población indígena en México: XI censo general de población y vivienda, Instituto Nacional de Estadística, Geografía e Informática, Instituto Nacional de Antropología e Historia y Universidad Nacional Autónoma de México, México.

Muñoz, H. (coord.), 1992, Población y sociedad en México, Miguel Ángel Porrúa, México.

Olivera, M., M. I. Ortiz y C. Valverde, 1992, La población y las lenguas indígenas de México en 1970, Universidad Nacional Autónoma de México, México.
Pujadas, J. J., 1993, Etnicidad: identidad cultural de los pueblos, eudema, Madrid.

Valdés, L. M. y M. T. Menéndez, 1987, Dinámica de la población de habla indígena: 1900-1980, Instituto Nacional de Antropología e Historia, México.

Valdés, L. M., 1989, El perfil demográfico de los indios mexicanos, Siglo xxi, México.

—, 2003, Los indios mexicanos en los censos del año 2000, Universidad Nacional Autónoma de México, México. www.conapo.gob.mx (Comisión Nacional de Población)

www.cdi.gob.mx (Comisión Nacional para el Desarrollo de los Pueblos Indígenas)

www.un.org (Naciones Unidas) 